\title{
Hypertension-associated C825T polymorphism impairs the function of G $\beta 3$ to target GRK2 ubiquitination
}

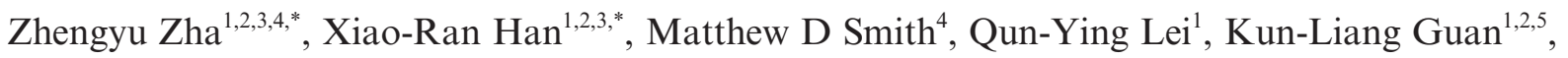 \\ Yue Xiong ${ }^{1,2,4}$ \\ ${ }^{1}$ Key Laboratory of Molecular Medicine, Ministry of Education, and Department of Biochemistry and Molecular Biology, Fudan \\ University Shanghai Medical College, Shanghai, China; ${ }^{2}$ Molecular and Cell Biology Lab, Institutes of Biomedical Sciences, \\ Fudan University, Shanghai, China, ${ }^{3}$ School of Life Sciences, Fudan University, Shanghai, China; ${ }^{4}$ Department of Biochemistry \\ and Biophysics, Lineberger Comprehensive Cancer Center, University of North Carolina at Chapel Hill, Chapel Hill, NC, USA; \\ ${ }^{5}$ Department of Pharmacology and Moores Cancer Center, University of California at San Diego, La Jolla, CA, USA
}

Population-based and case-control studies in different ethnicities have linked a polymorphism, C825T, in exon 10 of GNB3 gene to hypertension and several additional diseases. The 825T allele is associated with alternative splicing and

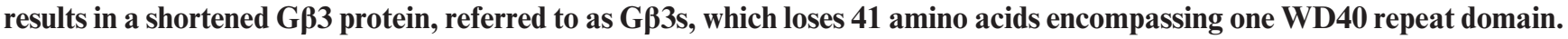
The mechanism of how GB3 C825T polymorphism is associated with hypertension has remained unclear, but an impairment

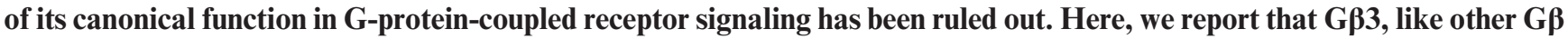
proteins, binds to DDB1 and assembles a DDB1-CUL4A-ROC1 E3 ubiquitin ligase (CRL4A ${ }^{\mathrm{G} \beta 3}$ ) to target GRK2

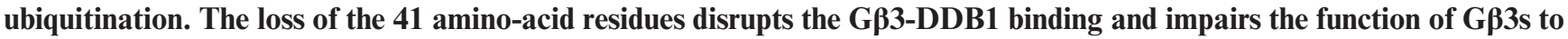
ubiquitinate GRK2. GRK2 ubiquitination levels were decreased and protein levels were accumulated in the blood samples of Gß3 825T allele carriers. Deletion of $\mathrm{Cul4a}$ in mice resulted in systolic pressure increased and weakened heart function in male mice that can be partially rescued by the deletion of one $G r k 2$ allele. These results reveal a mechanism explaining the link between Gß3 C825T polymorphism and hypertension.

Cell Discovery (2016) 2, 16005; doi:10.1038/celldisc.2016.5; published online 26 April 2016

\section{Introduction}

G-protein-coupled receptors (GPCRs) comprise the largest known family of cell-surface receptors, regulate numerous physiological processes and have a major role in medicine with $\sim 30 \%$ of current therapeutics targeting these seven transmembrane receptors $[1,2]$. Canonical signals from GPCRs are commonly relayed to intracellular effector proteins by trimeric $\mathrm{G}$ proteins,

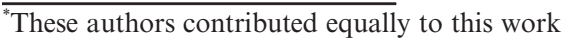
Correspondence: Q-Y Lei

Tel: +86-21-54237935; Fax: +86-21-54237853;

E-mail: qlei@fudan.edu.cn

or KL Guan

Tel: +858-531-3821; Fax: +86-21-54237450;

E-mail: kuguan@ucsd.edu

or Y Xiong

Tel: +919-962-2142; Fax: +919-966-8799;

E-mail: yxiong@email.unc.edu

Received 24 November 2015; accepted 8 February 2016
}

composed of an $\alpha, \beta$ and $\gamma$ subunit (G $\alpha \beta \gamma)$ [3]. Impaired GPCR signaling is linked with a wide range of human diseases, including heart disease, cancer and inflammation [4-6]. The G-protein beta 3 subunit (GNB3) C825T polymorphism was detected through a candidate gene approach using cell lines with enhanced G-protein activation from patients with essential hypertension [7]. Independent studies have confirmed an association of the $825 \mathrm{~T}$ allele with hypertension in Caucasians [8]. Multiple subsequent population-based and case-control studies in different ethnicities have supported the association between this polymorphism with hypertension, as well as obesity and atherosclerosis, but the mechanism underlying the $825 \mathrm{~T}$ polymorphism remains unclear [8-12]. The $825 \mathrm{~T}$ allele in exon 10 causes alternative splicing that deletes 123 bp in exon 9, resulting in an in-frame deletion of 41 amino-acid residues and a shortened splice variant of the $G \beta 3$, referred to as G $\beta 3$ s [7] (see diagram in Figure 1a). G $\beta 3$ s still binds to $G \alpha$ and $G \gamma$, and also has 


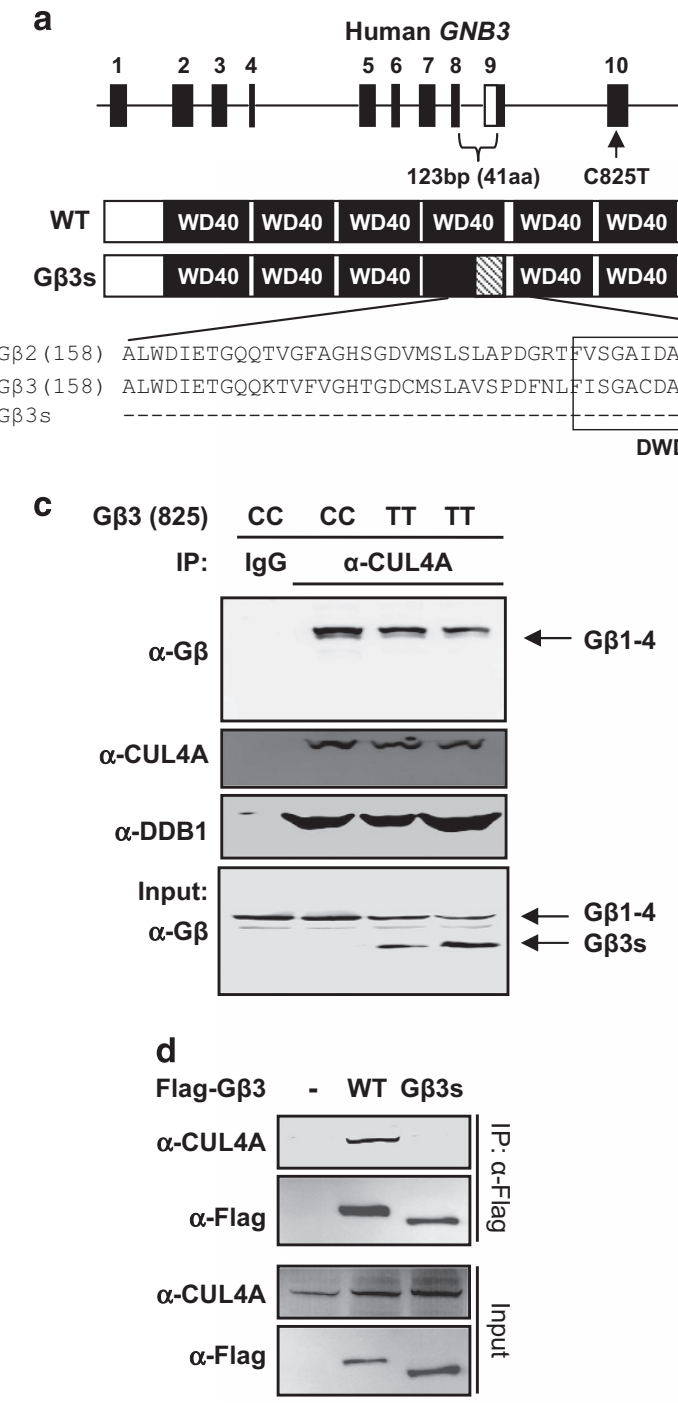

b
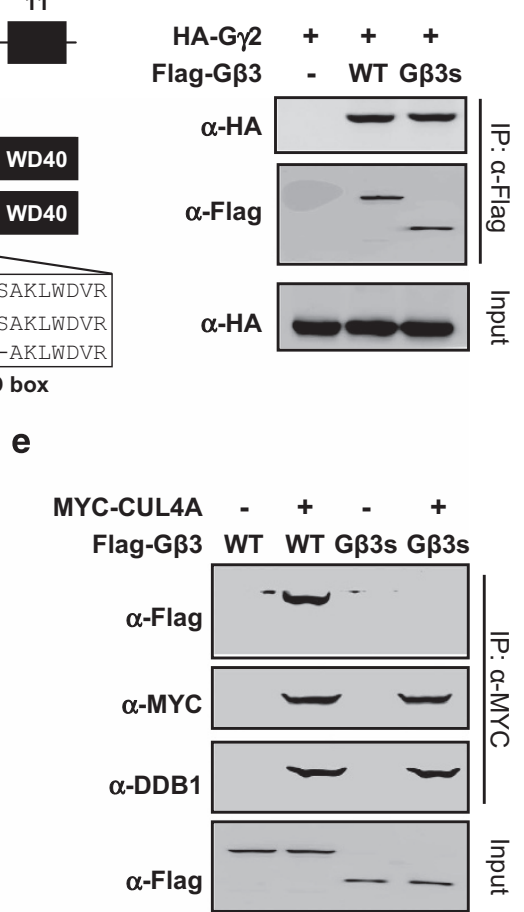

f Flag-G $\beta 3-+++$

ISO (10uM, h) $\quad-\quad 0 \quad 1 \quad 2$

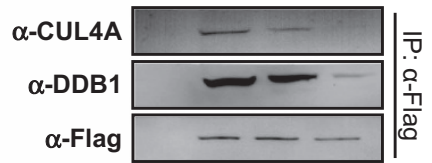

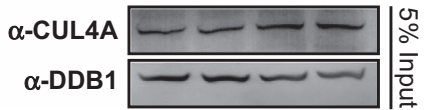

Figure 1 C825T-associated G $\beta 3$ s disrupts its binding to CUL4A-DDB1. (a) Schematic diagram of genome structure and protein domains of human $\mathrm{G} \beta 3$. The C825T is located in the exon 10 and the T allele is associated with splicing variant, G $\beta 3 \mathrm{~s}$, which has deleted 123 bp in exons 8 and 9, and an in-frame deletion of 41 amino-acid residues. (b) Both G $\beta 3$ and G $\beta 3$ s bind to G $\gamma 2$. HEK293 cells were co-transfected with plasmid expressing indicated proteins. Protein-protein binding was determined by co-immunoprecipitation (co-IP). (' $\alpha$-Flag' means anti-Flag antibody, ' $5 \%$ input' means $5 \%$ total protein for IP experiments were loaded, the same below). (c) CUL4A-DDB1 binds to endogenous $G \beta$, but not G $\beta 3 \mathrm{~s}$. A measure of $2 \mathrm{ml}$ of total blood samples from different $G \beta 3825$ allele carried in hypertensive patients was used to immunoprecipitate and was analyzed by immunoblotting. (d, e) Wild-type G $\beta 3$, but not disease-associated G $\beta 3$ s variant, binds to CUL4A. HEK293 cells were transfected with plasmids expressing indicated proteins and protein-protein binding was determined by co-IP. (f) Isoproterenol (ISO) decreases CUL4A-DDB1-G $\beta 3$ binding. HEK293 cells were transfected with plasmids expressing Flag-G $\beta 3$ and then treated with ISO $(10 \mu \mathrm{m})$ for the indicated lengths of time. The levels of individual proteins and the protein-protein interaction was determined by co-IP and immunoblotting using the antibodies indicated.

activity in intracellular signal transduction $[3,7,8,10]$. Recently, we reported a non-canonical function of $G \beta$ subunits in GPCR signaling that G $\beta 2$ binds to CUL4A-DDB1 and assembles an E3 ubiquitin ligase complex, CRL4 ${ }^{\mathrm{G} \beta 2}$ (the substrate-recruiter DWD protein $G \beta$ is superscripted), to promote the ubiquitination and degradation of GRK2 [13], a member of
G-protein-coupled receptor kinases (GRKs, also known as $\beta$-adrenergic receptor kinase or $\beta$-ARKs) [14].

GRK2 has an important role in the GPCR desensitization process [15], and abnormally elevated GRK2 protein level is linked with multiple pathological conditions in humans [16], including myocardial infarction 
[17], heart failure and portal hypertension [18]. G $\beta 2$ and other $\mathrm{G} \beta$ subunits are WD40 proteins and interact with DDB1 via a mechanism shared with other DDB1binding WD40 (DWD, also known as DCAF) proteins [19-22], including a conserved DWD box we previously defined [19]. Notably, the 41 residues lost in the G $\beta 3$ s variant locate in the fourth WD repeat and contain more than half of the DWD box (Figure 1a). This raises the possibility that the $825 \mathrm{~T}$ allele may impair the function of G $\beta 3$ s in assembly of CRL4 E3 ligase and targeting GRK2 ubiquitination. This study was directed to test this possibility.

\section{Results}

\section{C825T-associated G $\beta 3$ s disrupts its binding to CUL4A- DDB1}

We first confirmed the binding of $\mathrm{G} \beta 3$ and $\mathrm{G} \beta 3 \mathrm{~s}$ with G $\gamma 2$ (Figure 1b). We then examined the endogenous interaction of $G \beta 3$ and $G \beta 3$ s with different subunits of CRL4 complex in human primary blood samples from different GNB3 carried hypertensive patients: two $825 \mathrm{C}$ homozygous carriers and two $825 \mathrm{~T}$ homozygous carriers. Patients were considered to be hypertensive in these experiments based on a systolic blood pressure $>140 \mathrm{~mm} \mathrm{Hg}$ and diastolic pressure $>90 \mathrm{~mm} \mathrm{Hg}$. We used anti-CUL4A antibody that can co-immunoprecipitate multiple CRL4 components, including both DDB1 and different G $\beta$ subunits [13], and an anti-Pan $G \beta$ antibody that recognizes G $\beta 3$ s (Figure 1c) to confirm that G $\beta 3$ s was specifically expressed in $825 \mathrm{~T}$ carriers, but not in $825 \mathrm{C}$ carriers. Notably, G $\beta 3$ s was not detected in the CUL4A immunocomplex when other $\mathrm{G} \beta$ proteins were readily detected. To confirm this result, we ectopically expressed Flag-tagged G $\beta 3$ and G $\beta 3$ s in HEK293 cells and examined their interaction with endogenous CUL4A. Whereas Flag-G $\beta 3$ readily bound with endogenous CUL4A, we found that Flag-G $\beta 3$ s did not (Figure 1d). Similar results were obtained using ectopically expressed MYC-tagged CUL4A and Flagtagged $G \beta 3 / G \beta 3$ s, which showed that $G \beta 3$, but not G $\beta 3$ s, bound with CUL4A-DDB1 (Figure 1e). Isoproterenol, a drug used clinically for its inotropic and chronotropic effects on the heart and as a sympathomimetic $\beta$-AR agonist, reduces the association of G $\beta 2$ with DDB1-CUL4A [13]. We found that treatment of HEK293 cells with isoproterenol also effectively reduced the association of G $\beta 3$ with CUL4A (Figure 1f), suggesting that G $\beta 3$-DDB1/CUL4A association, such as the G $\beta 2$-DDB1/CUL4A complex, was regulated by GPCR signaling. Together, these results indicate that G $\beta 3$ interacts with DDB1-CUL4A, that this regulation is subjected to the regulation by GPCR signaling and that G $\beta 3$ s resulting from disease-linked C825T polymorphism impairs its binding to CUL4ADDB1.

\section{G $\beta 3$ s loses the function to target GRK2 ubiquitination and stabilizes GRK2}

The main function of the association of $G \beta 2$ with DDB1-CUL4A is to form the CRL4 E3 ligase complex $\left(\mathrm{CRL} 4 \mathrm{~A}^{\mathrm{G} \beta 2}\right)$ and to ubiquitinate GRK2 [13]. When assayed directly by expression and co-immunoprecipitation, GRK2 was able to bind to both wild-type G $\beta 3$ and G $\beta 3$ s (Figure 2a). To determine whether G $\beta 3$ and G $\beta 3$ s could regulate the level of GRK2 ubiquitination, G $\beta 3$ or G $\beta 3$ s was overexpressed in HEK293 cells, and then the ubiquitination of endogenous GRK2 was determined. The ubiquitination of endogenous GRK2 protein was readily detected and significantly enhanced by the expression of $G \beta 2$ or $G \beta 3$, but not G $\beta 3$ s (Figure $2 b$ ). We noted that ubiquitinated GRK2 levels in cells expressing G $\beta 3$ s were even lower than untransfected cells, suggesting a dominant-negative inhibition of endogenous $G \beta 3$ by the CUL4A/DDB1-binding-deficient G $\beta 3$ s. An in vitro ubiquitination assay showed that incubation of immunopurified GRK2 with immunopurified CUL4A and G $\beta 3$ complexes resulted in robust GRK2 ubiquitination in the presence of E1, E2, ATP and ubiquitin (Figure 2c). GRK2 ubiquitination bands were not observed in the absence of G $\beta 3$ (lane 1) or the substrate GRK2 (lane 4), as they were only detected in the presence of G $\beta 3$ (lane 2), but not G $\beta 3$ s (lane 3). Together, these in vivo and in vitro ubiquitination assays demonstrate that $G \beta 3$, like $G \beta 2$, also targets GRK2 for ubiquitination and that this activity is disrupted in G $\beta 3$ s.

Endogenous GRK2 ubiquitination levels in human blood samples from individuals with GNB3 $825 \mathrm{C}$ or $825 \mathrm{~T}$ allele were also examined. Anti-GRK2 beads were used to pull-down endogenous GRK2 protein of blood samples from patients with hypertension, and an anti-ubiquitin antibody was used to detect the endogenous GRK2 ubiquitination level. GRK2 ubiquitination levels were significantly decreased in GNB3 $825 \mathrm{~T}$ allele samples when compared with those from samples with $825 \mathrm{C}$ homozygous or $825 \mathrm{C} / \mathrm{T}$ heterozygous alleles (Figure 2d).

We next examined whether $G \beta 3$ s regulates the stability of GRK2 protein and found that GRK2 is a relatively unstable protein with an estimated half-life $\left(t_{1 / 2}\right)$ of $\sim 2.3 \mathrm{~h}$ in cells expressing wild-type $\mathrm{G} \beta 3$ (Figure 2e). Ectopic expression of G $\beta 3$ s significantly increased the half-life of GRK2 beyond the 
a

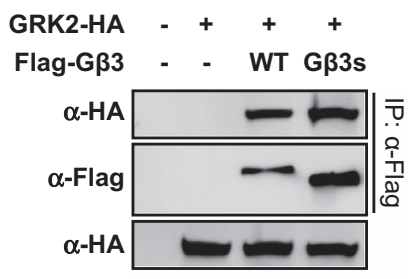

b

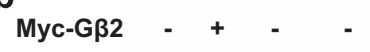

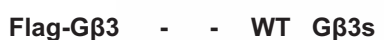

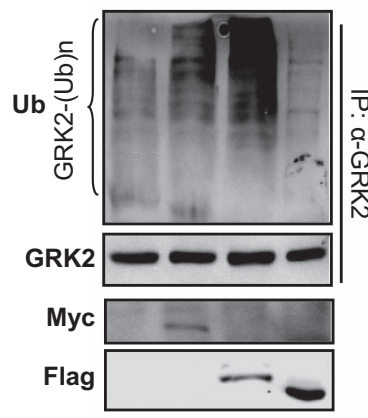

e

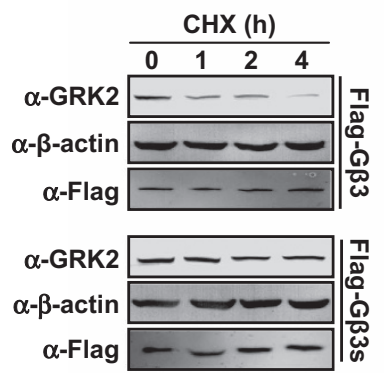

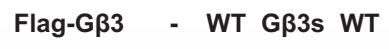

Myc-CUL4A ++++ HA-GRK2 + + + -

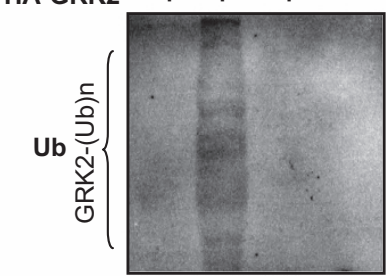
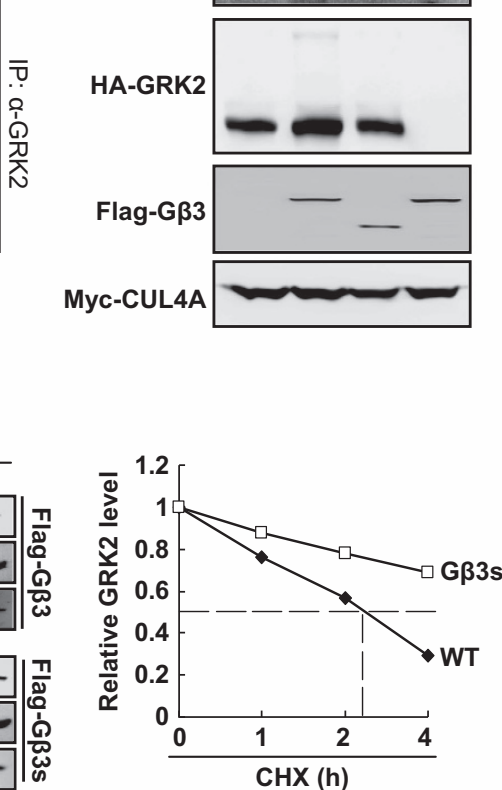

d

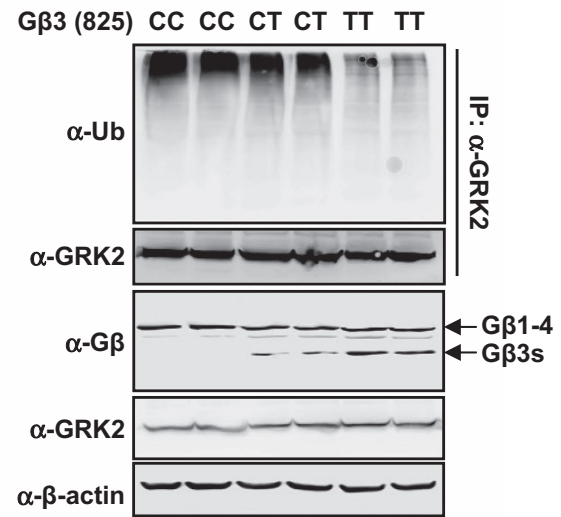

f

Normal

G 33 (825) CC CC CC CCCC CT CT CT CT CT TT TT TT TT GRK2/actin $1.0 \quad 0.81 .0 \quad 0.91 .11 .41 .21 .3 \quad 1.01 .11 .31 .31 .41 .4$

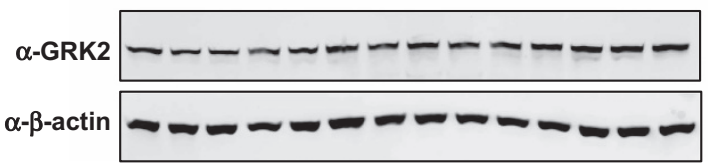

g

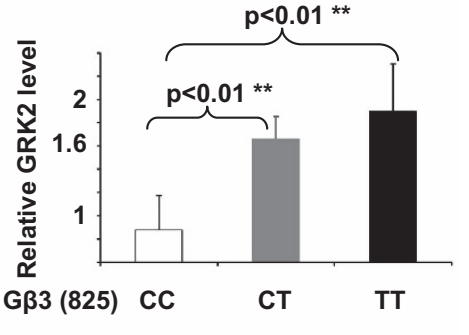

Hypertension

CC CC CC CC CT CT CT CT CT TT TT TT TT $\begin{array}{lllllllllllll}0.5 & 0.8 & 1.2 & 1.0 & 1.8 & 1.6 & 1.4 & 1.6 & 1.9 & 2.5 & 1.7 & 1.8 & 1.6\end{array}$

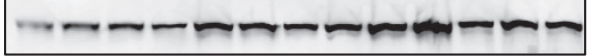

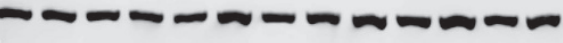

Figure 2 G $\beta 3$ s loses the ability to target GRK2 ubiquitination and stabilizes GRK2. (a) Both G $\beta 3$ and G $\beta 3$ s bind to GRK2. HEK293 cells were transfected with plasmids expressing indicated proteins and protein-protein interaction was determined by co-IP. (b) G $\beta 3$, but not G $\beta 3$ s, promotes GRK2 ubiquitination. HEK293 cells were transfected with plasmids expressing indicated proteins. Endogenous GRK2 was immunoprecipitated and analyzed for ubiquitination by immunoblotting. (c) In vitro ubiquitination of GRK2 by CRL4 ${ }^{\mathrm{G} 3} \mathrm{E} 3$ ligase, G $\beta 3$ s loses the ability to target GRK2 ubiquitination. Purified GRK2 protein was incubated with CUL4A immune complex alone or with purified G $\beta 3 / G \beta 3$ s in the presence of E1, E2, ATP and ubiquitin. After termination, the reaction mixtures were resolved by SDS-polyacrylamide gel electrophoresis, followed by immunoblotting with indicated antibodies. (d) Endogenous GRK2 ubiquitination level decreases in GNB3 825 T allele samples. A measure of 2 ml of total blood samples from different hypertensive patients who were/were not G $\beta 3825 \mathrm{~T}$ allele carriers was used to immunoprecipitate and analyzed for ubiquitination by immunoblotting. (e) Wild-type G $\beta 3$, but not disease-associated G $\beta 3 \mathrm{~s}$ variant, promotes the destabilization of GRK2. HEK293 cells were transfected with plasmids expressing indicated proteins. The half-life of GRK2 protein was determined by $\mathrm{CHX}$ treatment for different lengths of time as indicated, followed by immunoblotting and quantification. (f) High GRK2 level is associated with G 33 s C825T polymorphism. A measure of 1 ml total blood samples from different G $\beta 3825$ allele carriers (left panel) or hypertensive patients (right panel) was used to for western blotting by the antibodies indicated. (g) Statistical analysis of GRK2 protein level in different GNB3 carriers. Comparisons between the two groups were performed with unpaired, two-tailed Student's $t$-test (Excel software). $P$-values $<0.01$ were marked by **. Data are presented as the mean \pm s.d. 
experimental duration ( $4 \mathrm{~h}$; Figure 2e). This suggests that overexpression of G $\beta 3$ s stabilizes GRK2 protein, a notion that is consistent with the finding that ectopic expression of $\mathrm{G} \beta 3 \mathrm{~s}$ also reduces the ubiquitination of endogenous GRK2 (Figure 2b).

The protein levels of endogenous GRK2 were determined in blood samples from both normal and hypertensive patients. We found that in normal patients, homozygous GNB3 $825 \mathrm{~T}$ was associated with higher levels of GRK2 compared with homozygous $825 \mathrm{C}$ or heterozygous $825 \mathrm{C} / \mathrm{T}$ (Figure $2 \mathrm{f}$, left panel). Likewise, GRK2 was also accumulated in GNB3 $825 \mathrm{~T}$ homozygote samples from hypertensive patients (Figure 2f, right panel). The differences in GRK2 protein steady-state levels between possessing the $\mathrm{CC}$, CT or TT genotype were significant $(P<0.01$; Figure $2 \mathrm{~g}$ ). These results further support the notion that G 33 C825T polymorphism is associated with increased GRK2 protein level and that the increase is not the consequence of, but may be exacerbated during hypertension.

\section{Male Cul4a ${ }^{-1-}$ mice develop hypertension that is partially rescued by loss of one Grk2 allele}

The findings that both G $\beta 2$ and G $\beta 3$ target GRK2 ubiquitination led us to seek genetic and physiological evidences supporting the function of CUL4A-DDB1G $\beta$ E3 ligase in the regulation of GRK2 in vivo. We previously characterized $\mathrm{Cul} 4 \mathrm{a}$ null mice and found that, although overall appearing to development normally, the male, but not female $\mathrm{Cul}_{4 a^{-1-}}$ mice develop cardiac hypertrophy [13]. Further analyses of blood pressures and cardiac functions were carried out, and the systolic blood pressures of 34 age-matched, 10-week-old male mice (11 wild-type mice, $8 \mathrm{Cul}_{4} \mathrm{a}^{+-}$ mice and $15 \mathrm{Cul}_{4} \mathrm{a}^{-1-}$ mice) were determined. Compared with wild-type mice, the systolic blood pressure of $\mathrm{Cul}_{4} \mathrm{a}^{-1-}$ male mice was significantly higher $(P<0.01$; Figure 3a). To determine whether the hypertension associated with $\mathrm{Cul} 4 \mathrm{a}$ deletion results in cardiac dysfunction, two-dimensional echocardiogram analysis was performed for male $\mathrm{Cul}_{4 \mathrm{a}^{-/-}}$and wild-type mice. $\mathrm{Cul}_{4 a^{-1-}}$ male mice displayed multiple parameters of cardiac dysfunction compared with wild types, including increased left ventricular (LV) volume (6.89 vs $11.2 \mu \mathrm{l}$ end-systolic volume in wild-type vs $\mathrm{Cul} 4 \mathrm{a}^{-1-}$ ) and decreased fractional shortening $\left(38.6 \pm 4.8 \%\right.$ vs $47.7 \pm 3.9 \%$ in wild type vs $\mathrm{Cul}_{4} \mathrm{a}^{-1-}$; Figure $3 b$ ).

The cardiac hypertrophy defect seen in $\mathrm{Cul}_{4} \mathrm{a}^{-/-}$mice can be partially rescued by deletion of one allele of Grk2 [13]. To demonstrate genetically that Grk2 mediates the function of Cul4a in maintaining blood pressure homeostasis and cardioprotection, we characterized blood pressure and cardiac function of $\mathrm{Cul} 4$; Grk2 double-mutant mice. Systolic blood pressure of $\mathrm{Cul}_{4 a^{-1-}} \mathrm{Grk2}^{+/-}$male mice was partially decreased compared with $\mathrm{CulHa}^{-1-}$ mice (Figure 3c). Echocardiogram analysis showed that although the $\mathrm{Cul} 4 \mathrm{a}^{-1-}$ male mice had weakened cardiac function $(\sim 70 \%$ ejection fraction and $65 \%$ fractional shortening compared with wild type), deletion of one Grk2 allele partially restored the heart function in $\mathrm{Cul}_{4} \mathrm{a}^{-1-} ; \mathrm{Grk}^{+/-}$ male mice $(\sim 120 \%$ ejection fraction and $125 \%$ fractional shortening; Figure 3d). Together, these molecular, cellular and physiological analyses establish that Cul4A-Gß-mediated GRK2 degradation has a direct and important role in the regulation of heart function in male mice and that an impairment of this function, such as those seen in C825 T carriers, may contribute to the development of hypertension and weakened heart function.

\section{Discussion}

In 1998, G $\beta 3$ C825T polymorphism was first reported to be associated with hypertension and was subsequently confirmed by multiple population-based or case-control studies in different ethnicities. It has since been extended to diseases directly linked with hypertension such as atherosclerosis, left ventricular hypertrophy, stroke and myocardial infarction, as well as additional diseases such as obesity, insulin resistance and depression [7, 8, 10-12, 23, 24]. The molecular mechanism underlying the change in G $\beta 3$ protein caused by C825T polymorphism, however, has remained unclear [25]. Deletion of 41 amino-acid residues resulting from the $825 \mathrm{~T}$ allele does not significantly impact the binding of $G \beta 3$ s to $G \alpha$ and $G \gamma$, nor the function for GDP/GTP exchange [7, 10], leading to the notion that the truncated $G \beta 3$ s remains biologically active, at least as a signal transducer for GPCR. Although one study reported that G $\beta 3$ s is unstable and fails to bind with either $\mathrm{G} \gamma$ or $\mathrm{G} \alpha$ [26], we found that $G \beta 3$ s appears to bind with $G \gamma 3$ indistinguishable from that of wild-type G $\beta 3$ (Figure 1b). Instead, we found that loss of the 41 residues abolished G $\beta 3$ s binding to DDB1. We demonstrated that both ectopically and endogenously expressed G $\beta 3$ s lost its ability to interact with DDB1 and the ability to assemble an active CRL4 E3 ligase complex. Supporting the disruptive effect on association with DDB1 and formation of CRL4 E3 complex by the deletion of 41 residues, we show that G $\beta 3$ s, although still capable 

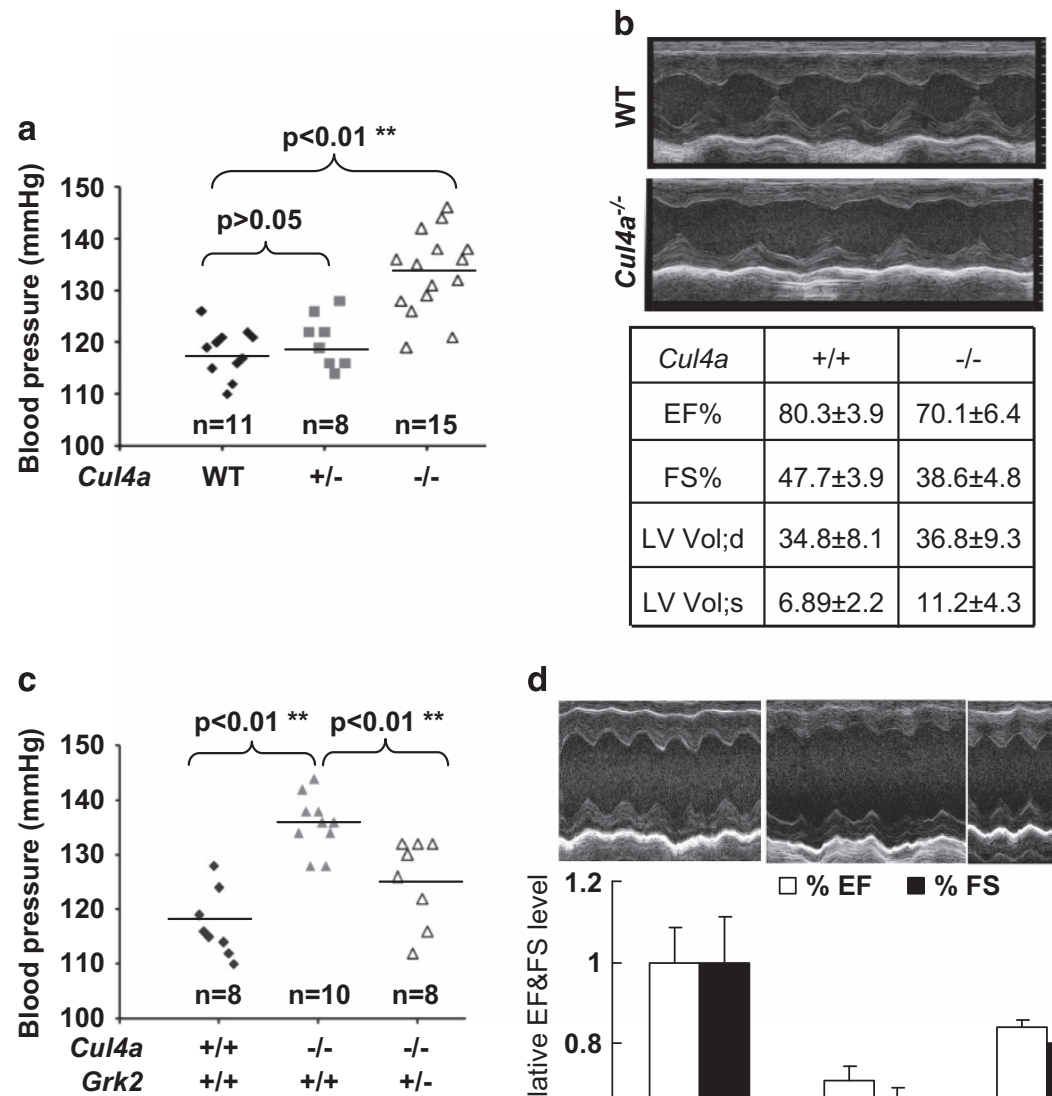

d

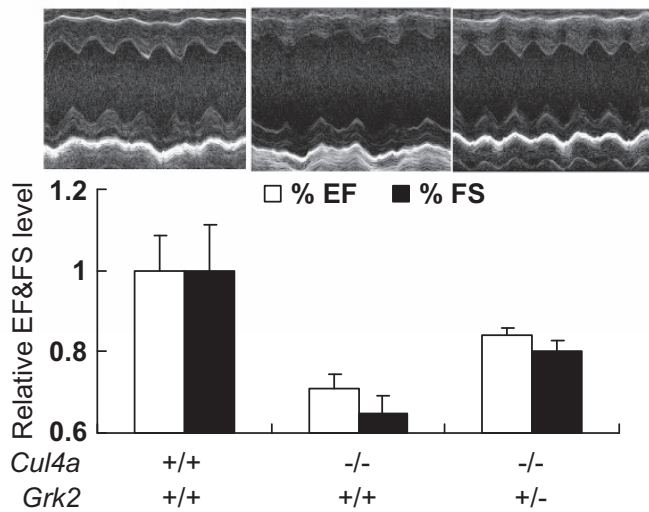

Figure 3 Male Cul4a $a^{-/-}$mice develop hypertension that is partially rescued by loss of one Grk2 allele. (a) Male Cul4a ${ }^{-/-}$mice develop hypertension. The systolic blood pressure (SBP) of 34 age-matched male mice of different genotypes was determined by NIBP mouse blood pressure meter machine. The statistical significances of heart weight differences between different genotypes were determined by $P$-value calculation as indicated. (b) $\mathrm{Cu} / 4 a^{-/-}$male mice have weaker heart function. The heart function of six pairs of littermate wild-type and $\mathrm{Cu}_{4} \mathrm{a}^{-/-}$male mice were determined by echocardiogram (ECHO) analysis. The values of heart ejection fraction (EF \%), fractional shortening (FS \%), end-diastolic (LV vol; d) and end-systolic (LV vol; s) of left ventricular were the average of six mice of each genotype. (c) Loss of one Grk2 allele partially rescues hypertension of Cul4a ${ }^{-/}$mice. The SBP of 26 age-matched male mice of different genotypes was determined. The statistical significance of heart weight differences between different genotypes was indicated by the $P$-value shown. (d) Loss of one Grk2 allele partially rescues weak heart function of $\mathrm{Cul}_{4} \mathrm{a}^{---}$mice. The heart function for different genotype male mice was determined by ECHO analysis (under isoflurane treatment). The values of heart ejection fraction (EF \%) and fractional shortening (FS \%) were compared with wildtype mice.

of binding to substrate GRK2, fails to catalyze GRK2 ubiquitination and promotes GRK2 stabilization and accumulation in cells. The pathological significance of an impairment of GRK2 degradation by the CRL4A ${ }^{\mathrm{G} \beta}$ E3 ligases, including CRL4A ${ }^{\mathrm{G} \beta 3}$, is supported by the finding that $\mathrm{Cul} 4 \mathrm{a}$ mutant mice accumulate higher levels of Grk2 protein and develop cardiac hypertrophy [26], hypertension and weakened heart function that can be partially rescued by the deletion of Grk2 (Figure 3). Although CUL4A can assemble potentially multiple E3 ligases including several CRL4A ${ }^{\mathrm{G} \beta}$ complexes, we suggest that loss of the ability to assemble
CRL4A ${ }^{\mathrm{G} \beta 3}$ complex and target GRK2 ubiquitination represents a major mechanism underlying the diseaseassociated C825T polymorphism.

\section{Materials and Methods}

\section{Cell culture and transfection}

HEK293 and HEK293T cells were cultured in Dulbecco's modified Eagle's medium supplemented with 10\% newborn calf serum, $100 \mathrm{U} \mathrm{ml}^{-1}$ penicillin and streptomycin (Gibco, Grand Island, NY, USA). Mouse embryonic fibroblast cells were maintained in Dulbecco's modified Eagle's medium 
supplemented with 10\% fetal calf serum (Gibco), 1\% L-glutamine, $100 \mathrm{U} \mathrm{ml}^{-1}$ penicillin and streptomycin. Cell transfection was performed using Lipofectamine 2000 (Life Technologies, Grand Island, NY, USA) or calcium phosphate method. Cells were collected at $36-48 \mathrm{~h}$ post transfection for protein analyses.

\section{Antibodies and immunological procedures}

Protein lysates were prepared by lysing HEK 293 cells in a buffer containing $50 \mathrm{~mm}$ Tris- $\mathrm{HCl}(\mathrm{pH} 7.5), 150 \mathrm{~mm} \mathrm{NaCl}, 0.1 \%$ SDS, $0.5 \%$ deoxycholate, $0.5 \%$ Nonidet P-40, $1 \mathrm{~mm}$ EDTA, $1 \mathrm{~mm}$ PMSF, $25 \mathrm{~mm} \mathrm{NaF}$ and a mixture of protease inhibitors. Cell lysate $(20 \mu \mathrm{g})$ was resolved by SDS-polyacrylamide gel electrophoresis, followed by western blotting analysis. Antibodies recognizing Flag (Sigma, St Louis, MO, USA), GRK2 (Santa Cruz, Dallas, TX, USA), HA (Santa Cruz), Myc (Santa Cruz), pan-G $\beta$ antibody (Santa Cruz) and $\beta$-actin (Cell Signaling, Shanghai, China) were purchased commercially. Antibodies to DDB1 and CUL4A have been described previously [27].

For immunoprecipitation experiments, $800 \mu \mathrm{g}$ of total protein in cell lysate was incubated with anti-Flag M2-agarose (Sigma) or anti-GRK2 beads (Santa Cruz) for $3 \mathrm{~h}$ at $4{ }^{\circ} \mathrm{C}$. Beads were washed three times with lysis buffer and centrifuged at $2000 \mathrm{~g}$ for $3 \mathrm{~min}$ between each wash. Protein was eluted from beads with $50 \mu \mathrm{l}$ of SDS sample buffer. Lysates were resolved on $8-15 \%$ SDS-polyacrylamide gel electrophoresis gels and transferred on to nitrocellulose (Bio-Rad, Hercules, CA, USA) for western blotting.

For experiments involving human blood, 2-5 $\mathrm{ml}$ of total blood samples was used for each immunoprecipitation experiment. The blood samples were centrifuged at $2000 \mathrm{~g}$ for $5 \mathrm{~min}$, and the precipitated blood cells were lysed in a buffer containing $50 \mathrm{~mm}$ Tris- $\mathrm{HCl}$ ( $\mathrm{pH} 7.5$ ), $150 \mathrm{~mm} \mathrm{NaCl}, 0.1 \%$ SDS, $0.5 \%$ deoxycholate, $0.5 \%$ Nonidet P-40, 1 mm EDTA, $1 \mathrm{~mm}$ PMSF, $25 \mathrm{~mm} \mathrm{NaF}$ and a mixture of protease inhibitors. The total protein from cell lysates was used for immunoprecipitation experiments, which were described above.

\section{In vitro ubiquitin ligation assays}

Plasmids expressing Myc-CUL4A, HA-GRK2, Flag-G $\beta 3$ or Flag-G $\beta 3$ s were individually transfected into $293 \mathrm{~T}$ cells by Lipofectamine 2000. At $48 \mathrm{~h}$ after transfection, cells were lysated into a buffer containing $50 \mathrm{~mm}$ Tris- $\mathrm{HCl}(\mathrm{pH} 7.5), 150 \mathrm{~mm}$ $\mathrm{NaCl}, 0.1 \%$ SDS and a cocktail of protease inhibitors, followed by immunoprecipitation using Myc or HA sepharose (Santa Cruz). Immunocomplexes were washed with the lysis buffer and eluted by Myc or HA antigen peptides. Immunopurified HAGRK2 protein was mixed with Myc-CUL4A and Myc3-G $\beta 2$ in a ubiquitin ligation buffer $(50 \mathrm{~mm}$ Tris- $\mathrm{HCl} / \mathrm{pH} 7.4,5 \mathrm{~mm}$ $\mathrm{MgCl}_{2}, 2 \mathrm{~mm} \mathrm{NaF}, 2 \mathrm{~mm}$ ATP, $10 \mathrm{~nm}$ okadaic acid, $0.6 \mathrm{~mm}$ dithiothreitol, $12 \mu \mathrm{g}$ of bovine ubiquitin, $1 \mu \mathrm{g}$ of ubiquitin (Sigma), 60 ng of E1 (E301, Boston Biochem, Cambridge, MA, USA), $500 \mathrm{ng}$ of E2 (human Ubc5c), final volume $=30 \mu \mathrm{l}$ ). The reaction was incubated at $37^{\circ} \mathrm{C}$ for $1 \mathrm{~h}$ on a rotator with slow shaking and then terminated by boiling at $95^{\circ} \mathrm{C}$ with SDS sample buffer for $10 \mathrm{~min}$ before SDS-polyacrylamide gel electrophoresis. GRK2 ubiquitination was examined by immunoblotting with either anti-ub or anti-HA antibody.

\section{Noninvasive mouse blood pressure measurement}

The blood pressure of 2-month-old male mice was measured using tail cuff plethysmography blood pressure systems (IITC Life Science, Woodland Hills, CA, USA). Six measurements were collected on each mouse, and the average was then calculated after excluding the lowest and highest readings.

\section{Echocardiographic analysis}

Cross-sectional, two-dimensional and Doppler transthoracic echocardiography was performed by experienced sonographers using a Vevo770 Imaging System (VisualSonics Inc, Toronto, ON, Canada). The chests of the male mice were shaved and treated with a chemical hair remover to reduce ultrasound attenuation. Heart rate and core temperature were continuously monitored. Mice were anesthetized with 1-2\% isofluorane. Images were stored in the Visual Sonics Imaging System for offline analysis. The values of heart ejection fraction (EF \%), fractional shortening (FS \%), end-diastolic (LV vol; d) and endsystolic ( $\mathrm{LV} \mathrm{vol;} \mathrm{s)} \mathrm{of} \mathrm{left} \mathrm{ventricular} \mathrm{were} \mathrm{the} \mathrm{average} \mathrm{of} \mathrm{six} \mathrm{mice}$ of each genotype.

\section{Statistical analysis}

Comparisons between the two groups were performed with unpaired, two-tailed Student's $t$-test (Excel software, Microsoft Corp., Shanghai, China). $P$-values $<0.05$ were considered statistically significant. Data are presented as the mean \pm s.d.

\section{Conflict of Interest}

The authors declare no conflict of interest.

\section{Acknowledgements}

We thank the members of the Fudan MCB and Xiong laboratories for discussions throughout this study, Brenda Temple of UNC RL Juliano Structural Bioinformatics Core facility for the molecular modeling, Bev Koller, Howard Rockman, Dennis Abraham, Kathleen Caron and John Sondek for discussions, and Cam Patterson for discussion and critical reading of the paper. This work was supported by Chinese Ministry of Sciences and Technology 973 (grant number 2015CB910401), NSFC (grant numbers 81430057, 81225016 and 31271454), Shanghai Key basic research program (12JC1401100), Shanghai Outstanding Academic Leader (grant number 13XD1400600) and the Youth Science and Technology Leading Talent by MOST to QYL, NIH grants EY022611 and CA132809 (to K-LG), and GM067113 (to YX).

\section{References}

1 Rockman HA, Koch WJ, Lefkowitz RJ. Seventransmembrane-spanning receptors and heart function. Nature 2002; 415: 206-212.

2 Lefkowitz RJ, Shenoy SK. Transduction of receptor signals by beta-arrestins. Science 2005; 308: 512-517. 
3 Siderovski DP, Kimple AJ, Willard FS Large G-proteins In: Begley TP. ed. Wiley Encyclopedia of Chemical Biology. Hoboken, NJ, USA: John Wiley \& Sons. 2009.

4 Dupre DJ, Robitaille M, Rebois RV, Hebert TE. The role of Gbetagamma subunits in the organization, assembly, and function of GPCR signaling complexes. Аnпи Rev Pharmacol Toxicol 2009; 49: 31-56.

5 Faruqi MG. Protein-coupled receptors: expanding the detection of GPCR activation. Nat Rev Drug Discov 2012; 11: 830

6 Stevens RC, Cherezov V, Katritch V et al. The GPCR Network: a large-scale collaboration to determine human GPCR structure and function. Nat Rev Drug Discov 2013; 12: 25-34.

7 Siffert W, Rosskopf D, Siffert G et al. Association of a human G-protein beta3 subunit variant with hypertension. Nat Genet 1998; 18: 45-48.

8 Benjafield AV, Jeyasingam CL, Nyholt DR, Griffiths LR, Morris BJ. G-protein beta3 subunit gene (GNB3) variant in causation of essential hypertension. Hypertension 1998; 32: 1094-1097.

9 Siffert W, Forster P, Jockel KH et al. Worldwide ethnic distribution of the $\mathrm{G}$ protein beta 3 subunit $825 \mathrm{~T}$ allele and its association with obesity in Caucasian, Chinese, and Black African individuals. J Am Soc Nephrol 1999; 10: 1921-1930.

10 Dobrev D, Wettwer E, Himmel HM et al. G-Protein beta (3)-subunit $825 \mathrm{~T}$ allele is associated with enhanced human atrial inward rectifier potassium currents. Circulation 2000; 102: 692-697.

11 Kato N, Sugiyama T, Morita H, Kurihara H, Yamori Y, Yazaki Y. G protein beta3 subunit variant and essential hypertension in Japanese. Hypertension 1998; 32: 935-938.

12 Schunkert H, Hense HW, Doring A, Riegger GA, Siffert W. Association between a polymorphism in the G protein beta3 subunit gene and lower renin and elevated diastolic blood pressure levels. Hypertension 1998; 32: $510-513$.

13 Zha Z, Han X, Smith Matthew D et al. A non-canonical function of $\mathrm{G} \beta$ as a subunit of $\mathrm{E} 3$ ligase in targeting GRK2 ubiquitination. Mol Cell 2015; 58: 794-803.

14 Mayor F Jr., Lucas E, Jurado-Pueyo M et al. G Proteincoupled receptor kinase 2 (GRK2): a novel modulator of insulin resistance. Arch Physiol Biochem 2011; 117: 125-130.

15 Lodowski DT, Pitcher JA, Capel WD, Lefkowitz RJ, Tesmer JJ. Keeping G proteins at bay: a complex between $\mathrm{G}$ protein-coupled receptor kinase 2 and Gbetagamma. Science 2003; 300: 1256-1262.

16 Gurevich EV, Tesmer JJ, Mushegian A, Gurevich VV. G protein-coupled receptor kinases: more than just kinases and not only for GPCRs. Pharmacol Ther 2012; 133: 40-69.

17 Yu X, Huang S, Patterson E et al. Proteasome degradation of GRK2 during ischemia and ventricular tachyarrhythmias in a canine model of myocardial infarction. Am J Physiol Heart Circ Physiol 2005; 289: H1960-H1967.

18 Liu S, Premont RT, Kontos CD, Zhu S, Rockey DC. A crucial role for GRK2 in regulation of endothelial cell nitric oxide synthase function in portal hypertension. Nat Med 2005; 11: 952-958.

19 He YJ, McCall CM, Hu J, Zeng Y, Xiong Y. DDB1 functions as a linker to recruit receptor WD40 proteins to CUL4-ROC1 ubiquitin ligases. Genes Dev 2006; 20: 2949-2954.

20 Higa LA, Wu M, Ye T, Kobayashi R, Sun H, Zhang H. CUL4-DDB1 ubiquitin ligase interacts with multiple WD40-repeat proteins and regulates histone methylation. Nat Cell Biol 2006; 8: 1277-1283.

21 Jin J, Arias EE, Chen J, Harper JW, Walter JC. A family of diverse Cul4-Ddb1-interacting proteins includes Cdt2, which is required for $\mathrm{S}$ phase destruction of the replication factor Cdt1. Mol Cell 2006; 23: 709-721.

22 Angers S, Li T, Yi X, MacCoss MJ, Moon RT, Zheng N. Molecular architecture and assembly of the DDB1-CUL4A ubiquitin ligase machinery. Nature 2006; 443: 590-593.

23 Whalen EJ, Foster MW, Matsumoto A et al. Regulation of beta-adrenergic receptor signaling by S-nitrosylation of G-protein-coupled receptor kinase 2. Cell 2007; 129: $511-522$.

24 Klenke S, Kussmann M, Siffert W. The GNB3 C825T polymorphism as a pharmacogenetic marker in the treatment of hypertension, obesity, and depression. Pharmacogenet Genomics 2011; 21: 594-606.

25 Semplicini A, Grandi T, Sandona C, Cattelan A, Ceolotto G. G-Protein beta3-subunit gene C825T polymorphism and cardiovascular risk: an updated review. High Blood Press Cardiovasc Prev 2015; 22: 225-232.

26 Jiang B, Zhao W, Yuan J et al. Lack of Cul4b, an E3 ubiquitin ligase component, leads to embryonic lethality and abnormal placental development. PLoS One 2012; 7 : e37070.

$27 \mathrm{Hu}$ J, McCall CM, Ohta T, Xiong Y. Targeted ubiquitination of CDT1 by the DDB1-CUL4A-ROC1 ligase in response to DNA damage. Nat Cell Biol 2004; 6: 1003-1009.

This work is licensed under a Creative Commons Attribution 4.0 International License. The images or other third party material in this article are included in the article's Creative Commons license, unless indicated otherwise in the credit line; if the material is not included under the Creative Commons license, users will need to obtain permission from the license holder to reproduce the material. To view a copy of this license, visit http://creativecommons.org/licenses/by/4.0/ 